\title{
Particle Swarm Optimization (PSO) applied to Fuzzy Modeling in a Thermal-Vacuum System
}

\author{
Rogério Marinke $^{1 \mathrm{a}} \quad$ Ernesto Araujo $^{2} \quad$ Leandro dos Santos Coelho $^{3} \quad$ Ivone Matiko $^{1 \mathrm{~b}}$ \\ ${ }^{1}$ Faculdade de Tecnologia do Estado de São Paulo - FATEC, Brazil \\ armarinke@hotmail.com, ${ }^{b}$ prof_ivone@yahoo.com.br \\ ${ }^{2}$ Instituto Nacional de Pesquisas Espaciais - INPE, Brazil \\ Integration and Testing Laboratory - LIT \\ ernesto.araujo@lit.inpe.br \\ ${ }^{3}$ Pontificia Universidade Católica do Paraná-PUCPR, Brazil \\ Laboratory of Automation and Systems - LAS / PPGEPS \\ leandro.coelho@pucpr.br
}

\begin{abstract}
A nonlinear identification approach based on Particle Swarm Optimization (PSO) and TakagiSugeno (T-S) fuzzy model for describing dynamical behavior of a thermal-vacuum system is proposed in this paper. Identification of nonlinear systems is an important problem in engineering among what fuzzy models have received particular attention due to their potentialities to approximate nonlinear behavior. Meanwhile PSO is proposed as a method for optimizing the premise part of production rules, least mean squares technique is employed for consequent part of production rules of a T-S fuzzy model. Experimental application using a thermal-vacuum system, used for space environmental emulation and satellite qualification, is analyzed. Numerical results indicate that the PSO succeeded in constructing a T-S fuzzy model for nonlinear identification in this particular application.
\end{abstract}

\section{Introduction}

Nonlinear techniques using fuzzy modeling have received a great deal of attention and have been employed in many applications in system identification [1][2]. Takagi-Sugeno (T-S) fuzzy model, for instance, exhibits both high nonlinearity and simple structure [4][5]. The identification problem in T-S modeling consists of two major parts: structure identification and parameter identification. The structure identification is related to both the determination of the premise part and the consequent part of the production rules. It consists of determining the premise space partition and extracting the number of rules and determining the structure of the output elements (equations), respectively. Finally, the parameter-learning task consists of determining the system parameters, i.e., membership functions, so that a performance measure based on the output errors is minimized.

This paper addresses the question of obtaining a fuzzy T-S model through Particle Swarm Optimization (PSO) by employing data supplied by real-world industrial engineering problem. The hybrid PSO and fuzzy approach are used in a synergetic manner with great success in the field of computational intelligence, as mentioned in [6]-[9]. The structure identification of the premise and the consequent part of production rules of T-S fuzzy system are separately carried out in this paper by distinct methods. The T-S fuzzy system design employs particle swarm optimization [10] for figuring out the premise part, meanwhile, least mean squares is used for the calculus of consequent part of production rules of a T-S fuzzy system for nonlinear identification.

An experimental case study using a nonlinear thermal-vacuum system is analyzed by using the proposed design approach. Thermal-vacuum systems are employed mainly for emulating space environmental conditions and thus qualifying space systems, such as satellites, spacecrafts and so forth.

Requirements for the space sector establish that the controlled variable must be the temperature on the specimen surface but the original controller was 
designed to use the temperature on the shroud (set of pipes). Experts and operators use their experience and reasoning for operating such a system and, thus, suppress such a problem. An alternative for avoiding human failures and transforming the system automatic is both to mimic the human reasoning and to incorporate feedback control techniques. A fuzzy control approach was designed in order to emulate the human behavior and thus being able to cope with this sort of nonlinear, time-delay system [11][12]. While succeeding in doing so, this fuzzy control approach was designed through interviews and by try-and-error approach in order to model the knowledge and expertise of these specialists and operators. Alternatively, the fuzzy control design may be established automatically if there is a model for the thermal-vacuum chamber.

There are several optimization techniques for extracting fuzzy models by using data, such as artificial neural network (ANN), evolutionary genetic algorithm (GA), sequential quadratic program (SQP) and so on. This paper uses PSO for T-S fuzzy modeling in order to describe the dynamical behavior of the before mentioned thermal-vacuum system and so to explore the effectiveness of PSO in constructing a good T-S fuzzy model for nonlinear identification.

\section{Optimal Takagi-Sugeno Fuzzy Systems}

\subsection{Takagi-Sugeno Fuzzy Systems}

The identification of a T-S fuzzy model involves two primary tasks: parameter tuning and structure optimization. The parameter tuning procedure deals with the estimation of a feasible set of parameters for a given structure. The structure optimization procedure aims to find the optimal structure of the local models, the relevant premise variables and a suitable partition of the premise space.

The T-S models consist of linguistic IF-THEN rules that can be represented by the following general form:

$$
\begin{gathered}
R^{(j)}: I F z_{1} \text { IS A }_{1}^{j} \text { AND } \ldots \text { AND } z_{m} \text { IS A }_{m}^{j} \text { THEN } \\
g_{j}=w_{0}^{j}+w_{1}^{j} u_{1}^{j}+\ldots+w_{q j}^{j} u_{q j}^{j}
\end{gathered} .
$$

The IF statements define the premise part while the THEN functions constitute the consequent part of the fuzzy system; $\underline{z}=\left[z_{1}, \ldots, z_{m}\right]^{T}, i=1, \ldots, m$, is the input vector of the premise $p$, and $A_{i}^{j}$ are labels of fuzzy sets. The parameters $\underline{u}=\left[u_{1}^{j}, \ldots, u_{q j}^{j}\right]^{T}$ represents the input vector to the consequent part of $R^{(j)}$ that comprising $q_{j}$ terms; $g_{j}=g_{j}\left(u^{j}\right)$ denotes the $j$-th rule output which is a linear polynomial of the consequent input terms $u_{i}^{j}$, and $\underline{w}^{j}=\left[w_{0}^{j}, w_{1}^{j} \ldots, w_{q j}^{j}\right]^{T}$ are the polynomial coefficients that form the consequent parameter set. Each linguistic label $A_{i}^{j}$ is associated with a Gaussian membership function, $\mu_{A_{i}^{j}}\left(z_{i}\right)$ described by (2) where $m_{i j}$ and $\sigma_{i j}$ are, respectively, the mean value and the standard deviations of the Gaussian membership function:

$$
\mu_{A_{i}^{j}}\left(z_{i}\right)=\exp \left[-\frac{1}{2} \frac{\left(z_{i}-m_{i j}\right)^{2}}{\sigma_{i j}^{2}}\right] .
$$

The union of all these parameters formulates the set of premise parameters. The firing strength of rule $R^{(j)}$ represents its excitation level and it is given by:

$$
\mu_{j}(\underline{z})=\mu_{A_{1}^{j}}\left(z_{1}\right) \cdot \mu_{A_{2}^{j}}\left(z_{2}\right) \cdots \mu_{A_{m}^{j}}\left(z_{m}\right)
$$

The fuzzy sets pertaining to a rule form a fuzzy region (cluster) within the premise space, $A_{1}^{j} x A_{2}^{j} x \cdots x A_{m}^{j}$, with a membership distribution described by equation (3). Given the input vectors $z$ and $u^{j}, j=1, \ldots, M$, the final output of the fuzzy system is inferred by taking the weighted average of the local outputs $g_{j}\left(\underline{u}^{j}\right)$ that is given by

$$
y=\sum_{j=1}^{M} v_{j}(\underline{z}) \cdot g_{j}\left(\underline{u}^{j}\right)
$$

where $M$ denotes the number of rules and $v_{j}(z)$ is the normalized firing strength of $R^{(j)}$, which is defined as

$$
v_{j}(\underline{z})=\frac{\mu_{j}(\underline{z})}{\sum_{j=1}^{M} \mu_{j}(\underline{z})}
$$

The structure identification of T-S system is realized based on PSO for premise part optimization while the consequent part optimization is realized by batch least mean squares method [11].

\subsection{Particle Swarm Optimization (PSO) for Takagi-Sugeno (T-S) Fuzzy Modeling}

Particle Swarm Optimization (PSO) originally developed by Kennedy and Eberhart in 1995 is a population-based swarm algorithm [14][15]. Similarly to genetic algorithms [3], an evolutionary algorithm 
approach, PSO is an optimization tool based in a population where the position of each member/particle is a potential solution to an analyzed problem. Each particle in PSO has associated a randomized velocity that moves through the problem space. One advantage of PSO over genetic algorithms is that this method does not have operators, i.e., crossover and mutation. Moreover, PSO does not implement the survival of the fittest individuals; instead, it implements the simulation of social behavior.

Each particle in PSO keeps track of its coordinates in the problem space, which are associated with the best solution (fitness) it has achieved so far. This value is called pbest. Another "best" value that is tracked by the global version of the particle swarm optimizer is the overall best value. Its location, called gbest, is obtained indeed by any particle in the population. The past best position and the entire best overall position of the group are employed to minimize (maximize) the solution

The PSO concept consists of, at each time step, changing the velocity (acceleration) of each particle flying toward its pbest and gbest locations (global version of PSO). Acceleration is weighted by random terms, with separate random numbers being generated for acceleration toward pbest and gbest locations, respectively. The procedure ([16]-[18]) for implementing the global version of PSO is given by the following steps:

(i) Initialize a population (array) of particles with random positions and velocities in the $n$ dimensional problem space using uniform probability distribution function.

(ii) For each particle, evaluate its fitness value.

(iii) Compare each particle's fitness with the particle's pbest. If current value is better than pbest, then set pbest value equal to the current value and the pbest location equal to the current location in $n$ dimensional space.

(iv) Compare the fitness with the population's overall previous best. If current value is better than gbest, then reset gbest to the current particle's array index and value.

(v) Change the velocity, $v_{i}$, and position of the particle, $x_{i}$, according to equations (6) and (7):

$$
\begin{gathered}
\boldsymbol{v}_{\boldsymbol{i}}=w \cdot \boldsymbol{v}_{\boldsymbol{i}}+c_{1} \cdot u d() \cdot\left(\boldsymbol{p}_{\boldsymbol{i}}-\boldsymbol{x}_{\boldsymbol{i}}\right)+c_{2} \cdot U d() \cdot\left(\boldsymbol{p}_{\boldsymbol{g}}-x_{\boldsymbol{i}}\right) \\
\boldsymbol{x}_{i}=\left(\boldsymbol{x}_{i}+\Delta t \cdot \boldsymbol{v}_{i}\right) .
\end{gathered}
$$

(vi) Return to step (ii) until a stop criterion is met, usually a sufficiently good fitness or a maximum number of iterations (generations).

In this approach $x_{i}=\left[x_{i 1}, x_{i 2}, \cdots, x_{i n}\right]^{T}$ stands for the position and $v_{i}=\left[v_{i 1}, v_{i 2}, \cdots, v_{i n}\right]^{T}$ for the velocity of the $i$-th particle, and $p_{i}=\left[p_{i 1}, p_{i 2}, \cdots, p_{i n}\right]^{T}$ represents the best previous position of the $i$-th particle (the position giving the best fitness value). The first part in equation (6) is the momentum part of the particle. The second part is the 'cognition' one, which represents the independent thinking of the particle itself. Equation (7) represents the position update, according to its previous position and speed, considering $\Delta t=1$.

The inertia weight $w$ represents the degree of the momentum of the particles. The use of variable $w$, inertia weight as proposed by Shi and Eberhart [19], is responsible for dynamically adjust the velocity of the particles. This parameter is accountable for balancing between local and global search, consequently, needing less or more iterations for the algorithm to converge. A small value of inertia weight implies in a local search; a high one leads to a global search, yet with a high computational cost. However, linear decreasing inertia function may also be used if it is interested in reduce the influence of past velocities during the optimization process.

The index $g$ represents the index of the best particle among all the particles in the group. Variables $u d()$ and $U d()$ are two random functions in the range $[0,1]$. Positive constants $c_{1}$ and $c_{2}$ are denominated, respectively, cognitive and social components. These are the acceleration constants, responsible for varying the particle speed towards pbest and gbest. Commonly used values for them are set equal 2 . The velocity of the $i$-th particles on each dimension is clamped to a maximum velocity $V_{\max }$. If the sum of accelerations would cause the velocity on that dimension to exceed $V_{\max }$, which is a parameter specified by the user, then the velocity on that dimension is limited to $V_{\max }$. The parameter, $V_{\max }$, is used to determine the resolution with which the regions around the current solutions are searched. If $V_{\max }$ is too high, the PSO facilitates global search, and particles might fly past good solutions; if is too small, the PSO facilitates local search, and particles may not explore sufficiently beyond locally good regions. Experience says that $V_{\max }$ might be set to $20 \%$ of the dynamic range of the variable on each dimension.

\section{Thermal-vacuum System Identification}

\subsection{Problem Statement}

A thermal-vacuum system employed to reproduce expected space post-launch environments conditions 
for qualifying space systems consists of a chamber, a set of tubes (shroud) - from where heat and cold are transmitted through radiation -, and other auxiliary devices (Figure 1). The operation of the thermal shroud is achieved by means of a re-circulating, dense, and gaseous nitrogen (GN2) system. Resistance type heaters provide heat as required. Cooling the circulating gas stream is accomplished by spraying liquid nitrogen (LN2) into the circuit [20].

This system is not only nonlinear [21] but presents time-delay, is time-varying, and works in diverse operational conditions defined by various levels of temperature (set points) used during the test. For illustrate these characteristics, a dynamical response for a passive load is shown in Figure 2. Continuous and dashed lines represent input and output data, respectively. It is possible to notice, for instance, the chamber-load set presents as many heating and cooling rates as the operational conditions.

In a previous work, a fuzzy controller was designed to work as a supervisory-control mechanism in helping specialists to operate the thermal-vacuum chamber. Results and the suggested FRGS control approach are available in [11]-[13]. An alternative to improve the fuzzy controller performance is to find out a fuzzy model for representing the thermal dynamical behavior and thus to use it to tune the fuzzy control system. Here, PSO and fuzzy approaches work in a complementary and synergistic manner by using experimental data.

\subsection{Identification of T-S PSO-Fuzzy model}

The first part of data shown in Figure 2 was employed to elicit the fuzzy model through PSO. The first 800 initial data samples were discharged because they have constant values and would interfere in the identification process. The remaining data were used to validate the results.

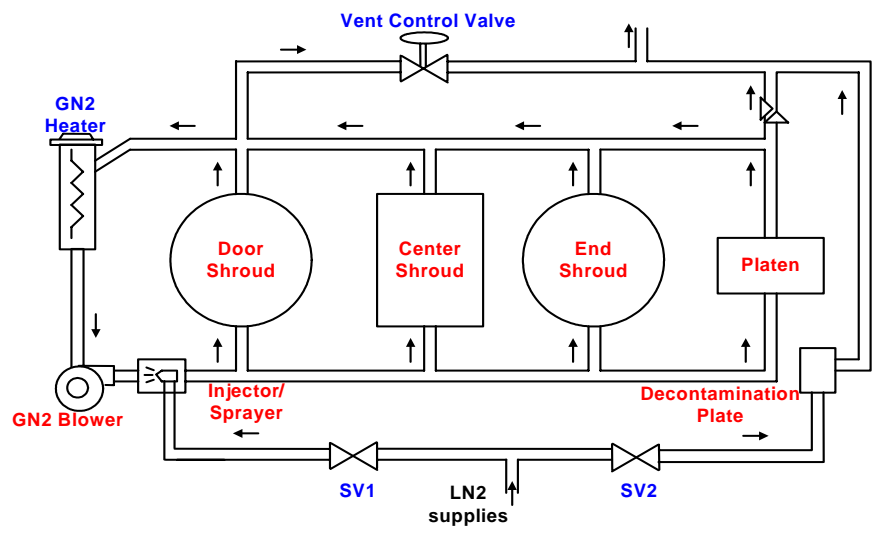

Figure 1 - Thermal-vacuum chamber.

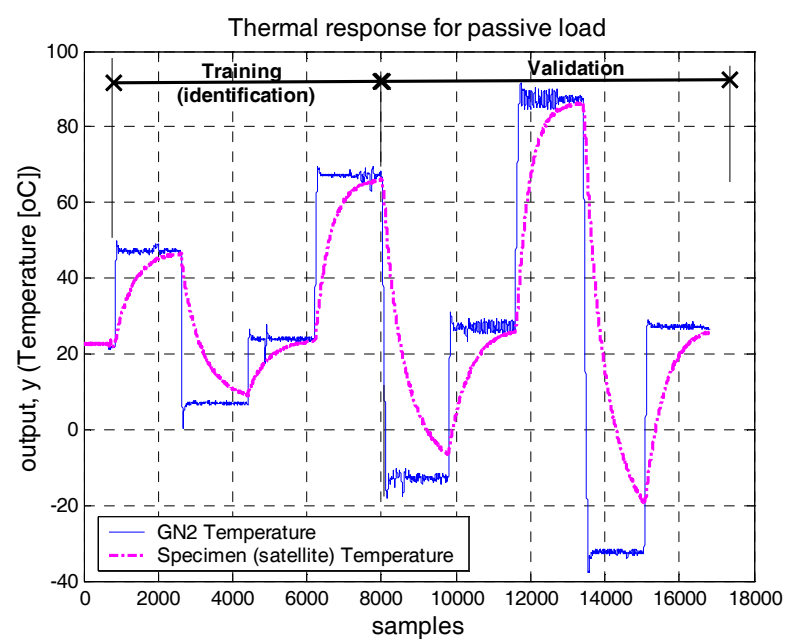

Figure 2 - Thermal response: passive load.

Although, PSO allows to extract the number of rules and to determine the premise and consequent elements, here this method is applied to obtain membership functions and thus to determine the premise space partition. In so doing, it uses a predefined number of rules and membership functions arbitrarily chosen for each input. Setting up this parameter as 3, PSO needs to deal with a vector whose elements are 9 centers and 3 spreads of a Gaussian membership function. Two parameters deeply related to the success of PSO are the amount and the initial position of particles. One of advantage of this technique is that the initial population of particles is randomly generated through an uniform distribution. The sufficient number of particles for this application was setup as 5. The main parameters of PSO approach are shown in Table 1.

The estimate PSO-Fuzzy model output, $\hat{y}(k)$, used for computing the minimum square error (8) when compared with the actual output, $y(k)$ was computed by using one-step ahead forecasting.

$$
\min _{\theta} \sum_{\mathrm{k}=1}^{\mathrm{N}}\|\hat{y}(k)-y(k)\|
$$

Among a population of potential solution to a problem, every particle has a fitness value for expressing appropriate optimization result. The function representing this quality measure employs the position of the particle, $x$, that is calculate after each iteration. In this paper, the fitness criteria chosen for evaluate the relationship between the real output and the estimate output during the optimization process was the maximization of Pearson multiple correlation coefficient. 
Table 1 - Parameters for PSO application

\begin{tabular}{l|c}
\hline \multicolumn{1}{c|}{ Parameter } & Selection \\
\hline Number of particles & 5 \\
\hline Number of iterations, $N$ & 30 \\
\hline Learning rate & $c_{1}=c_{2}=2.05$ \\
\hline Inertia weight & Linear decreasing (0.729 to 0.4) \\
\hline Optimization function & $\begin{array}{c}\text { Maximization of Pearson } \\
\text { Multiple correlation coefficient }\end{array}$ \\
\hline \hline
\end{tabular}

This coefficient gives the rate between the variability of two measures (variables) in which one is described by the variability of the other. The closer the Pearson coefficient is of unit, the more appropriate is the model. Suitable correlation factors considered for practical control applications are bigger than 0.9 and lower (or equal to) than 1.0 [22].

Distinct results were obtained during the optimization process according to the number of iterations employed. The fitness factor through time determined when this factor was set to 30 is shown in Figure 3. This optimization process was carried out several times and the best fitness value when using 30 iterations was 0.99835 as it is presented in Table 2. It is also possible to notice that the error rate between the fitness value for training and validation is 5,3\%. Two dynamical responses simulating the thermal-vacuum chamber when using T-S fuzzy models are shown in Figure 4. These models were obtained through Particle Swarm Optimization by using different sample rates of data. Continuous and dashed lines represents measured and simulated outputs. The experimental results had shown that the hybrid T-S fuzzy system and PSO approaches presented successful results due precision in predicting nonlinear dynamics.

Table 2 - PSO-Fuzzy fitness values

\begin{tabular}{c|c|c|c|c}
\hline \hline "n" & Fitness (max) & $\begin{array}{c}\text { Fitness } \\
\text { value } \\
\text { training) }\end{array}$ & $\begin{array}{c}\text { Fitness } \\
\text { Value } \\
\text { (validation) }\end{array}$ & $\begin{array}{c}\text { Error } \\
\text { Rate [\%] }\end{array}$ \\
\hline 01 & 0.000000 & 0.000000 & 0.000000 & $\mathrm{NaN}$ \\
\hline 02 & 0.831480 & 0.831480 & 0.000000 & 100 \\
\hline$\ldots$ & $\ldots$ & $\ldots$ & $\ldots$ & $\ldots$ \\
\hline 06 & 0.831480 & 0.831480 & 0.000000 & 100 \\
\hline 07 & 0.942170 & 0.942170 & 0.000000 & 100 \\
\hline 08 & 0.979684 & 0.979684 & 0.681561 & 30.4305 \\
\hline$\ldots$ & $\ldots$ & $\ldots$ & $\ldots$ & $\ldots$ \\
\hline 20 & 0.979684 & 0.979684 & 0.681561 & 30.4305 \\
\hline 21 & 0.985617 & 0.985617 & 0.906519 & 8.0252 \\
\hline$\ldots$ & $\ldots$ & $\ldots$ & $\ldots$ & $\ldots$ \\
\hline 23 & 0.985617 & 0.985617 & 0.906519 & 8.0252 \\
\hline 24 & 0.993551 & 0.993551 & 0.886662 & 10.7583 \\
\hline$\ldots$ & $\ldots$ & $\ldots$ & $\ldots$ & $\ldots$ \\
\hline 29 & 0.993551 & 0.993551 & 0.886662 & 10.7583 \\
\hline 30 & 0.998350 & 0.998350 & 0.944749 & 5.3690 \\
\hline \hline
\end{tabular}

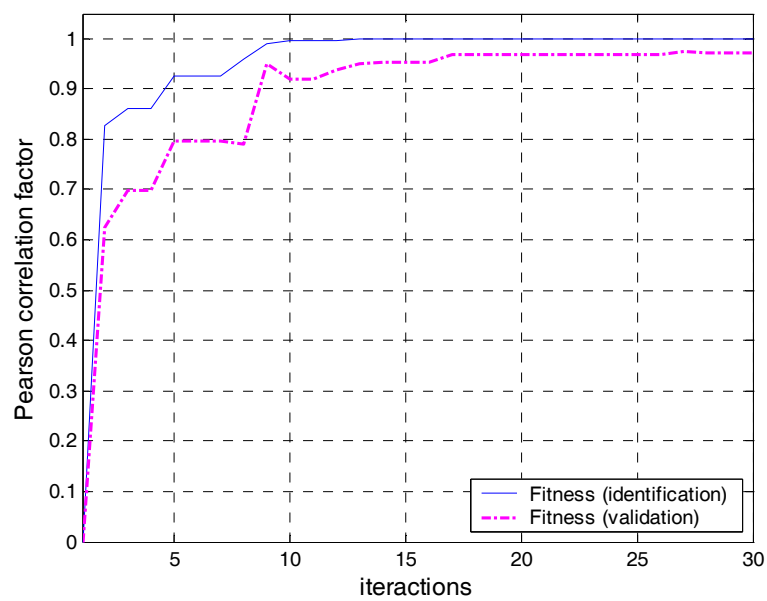

Figure 3 - Fitness estimation and validation

\section{Conclusion and Future Research}

In this work is presented a hyperspace search mechanism known as particle swarm optimization, PSO, to find out a T-S fuzzy model for a thermalvacuum system.

This approach presents several advantages, such as, initial population of particles is randomly generated through uniform distribution, there are no operators such as crossover and mutation in genetic algorithm, simple rules describe complex behavior, and there are simple code and low computational cost.

The elicited fuzzy model with only three membership functions determining the premise space partition demonstrated its effectiveness in emulating the time response for the thermal-vacuum system. Future work must be carried out to verify the influence of other parameters in obtaining the model. For example, the number of membership functions may be increased, and since there is large amount of data other step ahead forecasting modes may be exploited.

Since this paper accomplished its goal of checking the use of hybrid mechanisms for modeling a realworld industrial engineering problem, future work also includes a comparative approach with other learning mechanisms and/or optimization methods for generating T-S fuzzy models as well as with classical methods of identification through the use of statistical analysis.

\section{References}

[1] M.L. Hadjili, V. Wertz, "Takagi-Sugeno fuzzy modelling incorporating input variables selection", IEEE Trans. on Fuzzy Systems, v.10, n.6, pp. 728-742, 2002. 
[2] W. Pedrycz, F. Gomide, An Introduction to Fuzzy Sets: Analysis and Design, MIT Press ed.: Cambridge. MA, 1998.

[3] B. Wu, X. Yu, "Fuzzy modelling and identification with genetic algorithm based learning", Fuzzy Sets and Systems, v.113, pp. 351-365, 2000.

[4] T. Takagi, M. Sugeno, "Fuzzy identification of systems and its applications to modeling and control", IEEE Trans. on Systems. Man and Cybernetics, v.15, n.1, pp. 116-132, 1985.

[5] M. Sugeno, G.T. Kang, "Structure identification of fuzzy model”, Fuzzy Sets and Systems, v.28, pp. 15-33, 1988.

[6] Y. Liu, X. Zhu, J. Zhang, S. Wang, "Application of particle swarm optimization algorithm for weighted fuzzy rule-based system," Proc. Annual Conference of IEEE Industrial Electronics Society, IECON, Busan, Korea, pp. 2188-2191.

[7] C.-F. Juang, Y.-C. Liou, "TSK-type recurrent fuzzy network design by the hybrid of genetic algorithm and particle swarm optimization," Proc. IEEE International Conference on Systems, Man, and Cybernetics, The Hague, The Netherlands, vol. 3, pp. 2314-2318, 2004.

[8] M. Ma, C.-G. Zhou, L.-B. Zhang, Q.-S. Dou, "Automatic fuzzy rule extraction based on particle swarm optimization," Proc. International Conference on Machine Learning and Cybernetics, Shanghai, China, vol. 4, pp. 2242-2245, 2004.

[9] A. A. A. Esmin, A.R. Aoki, G.L-. Torres, "Particle swarm optimization for fuzzy membership functions optimization," Proc. IEEE International Conference on Systems, Man, and Cybernetics, Hammamet, Tunisia, vol. 3, 6 pp., 2002.

[10] J.F. Kennedy, R.C. Eberhart, R.C. Shi, Swarm Intelligence, Morgan Kaufmann Pub. San Francisco, CA, 2001.

[11] J.E. Araujo, S.A. Sandri, E.E.N. Macau, "Fuzzy Reference Gain Scheduling control systems", Proc. NAFIPS, pp. 461-464, Atlanta, 2000.

[12] J.E. Araujo, S.A. Sandri, E.E.N. Macau, "A new class of adaptive fuzzy control system applied in industrial thermal vacuum process", Proc. IEEE-ETFA, v.1. pp. 426-431, France, 2001.

[13] J.E. Araujo, K.H. Kienitz, "Adaptive reference-driven decision-making process", Proc. FUZZ-IEEE, v.1, pp. 452-457, St. Louis, 2003.

[14] R.C. Eberhart, J. Kennedy, "A new optimizer using particle swarm theory", Proc. Int. Symposium on Micro Machine and Human Science, pp. 39-43, Japan, 1995.

[15] J. Kennedy, R.C. Eberhart, "Particle swarm optimization", Proc. IEEE Intern. Conf. on Neural Networks, Perth, Australia, pp. 1942-1948. 1995.

[16] R.A. Krohling, L.S. Coelho, Y. Shi, "Cooperative particle swarm optimization for robust control system design", World Conference on Soft Computing in Industrial Applications, Granada, Spain, On-line. http://site: decsai.ugr.es/wsc7. 2002.

[17] R.A. Krohling, F. Hoffmann, L.S. Coelho, "Coevolutionary particle swarm optimization for min-max problems using Gaussian distribution" Proc. Congress on Evolutionary Computation. Portland. pp. 959-964, 2004.

[18] L.S. Coelho, R.A. Krohling, "Predictive controller tuning using modified particle swarm optimization based on Cauchy and Gaussian distribution", Soft Computing: Methodologies and Applications, Advances in Soft Computing Series, F. Hoffmann. M. Köppen. F. Klawonn. R. Roy (editors). Springer-Verlag. UK.

[19] Y. Shi and R.C. Eberhart. "Parameter selection in particle swarm optimizer." Proc. Annual Conf. on Evolutionary Programming. V.W. Porto. N. Saravan. D. Waagen. A. E. Eiben (editors). Berlin: Springer-Verlag. pp. 591-601. 1998.

[20] High Vacuum Systems Inc.. Operations and Maintenance Manual. Thermal Vacuum System with Thermally Conditioned Platen for Brazilian Space Research Institute. 1987.

[21] D.G. Gilmore, Satellite Thermal Control Handbook, The Aerospace Corporation Press., California, 1994.

[22] B. Schweizer, H. Xie, Y.C. Lee; "Fuzzy Logic models for ranking process effects"; IEEE Trans. on Fuzzy Systems, v.5, n.4, pp.545-55, 1997.
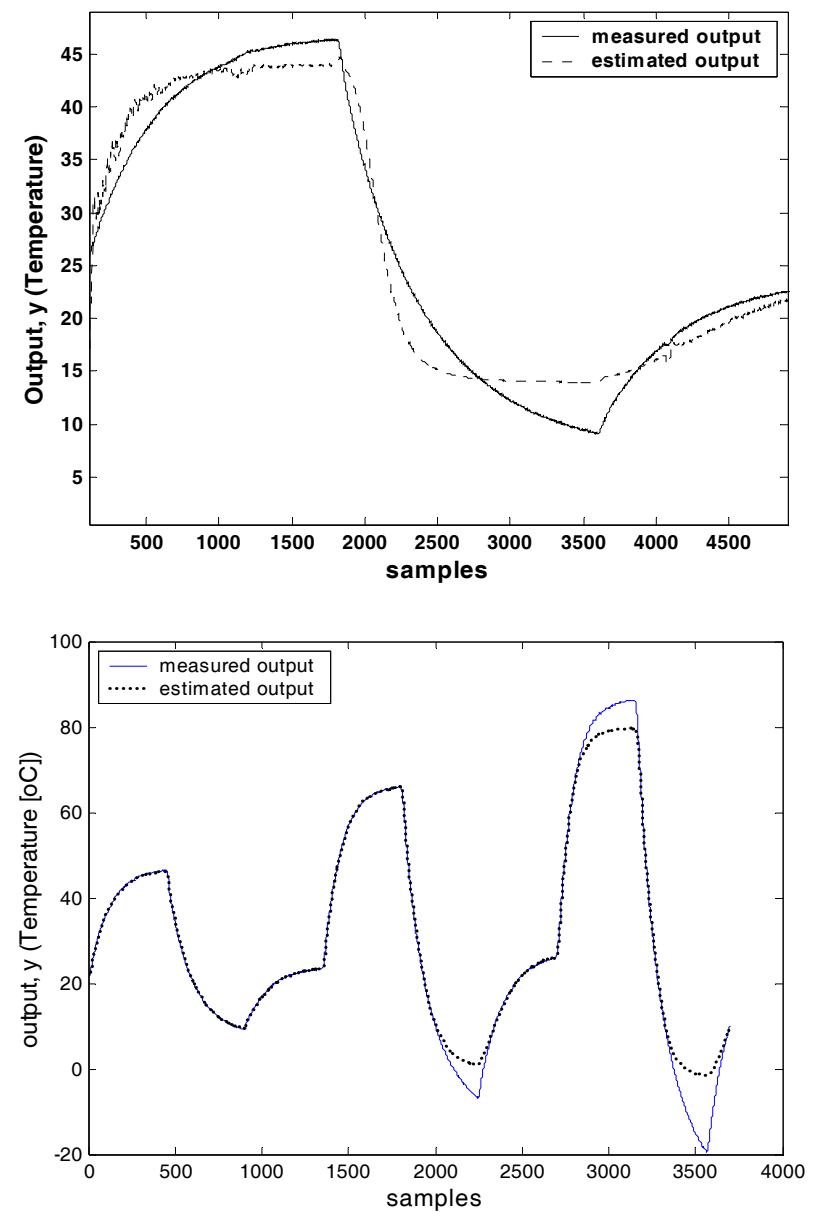

Figure 4 - Responses obtained through resulting T-S model. 